\title{
Determination of Mechanical Energy Loss in Steady Flow by Means of Dissipation Power
}

\author{
Wojciech Artichowicz, Jerzy M. Sawicki \\ Gdańsk University of Technology, Faculty of Civil and Environmental Engineering, ul. G. Narutowicza \\ 11/12, 80-233 Gdańsk, Poland, e-mails: wojartic@pg.edu.pl, jsaw@pg.edu.pl
}

(Received April 06, 2017, revised October 24, 2017)

\begin{abstract}
When systems of simple geometry like pipes or regular channels are considered, the mechanical energy loss of the fluid flow can be expressed by local and longitudinal empirical energy loss coefficients. However, in the case of large spatially distributed objects, there are no simple approaches to this task. In practice, general recommendations addressing different types of objects are used, but they usually provide very coarse estimates of energy loss.

In this work, a new methodology for determination of mechanical energy loss in steady flow is proposed. This methodology is based on the observation that the magnitude of the power of energy dissipation in turbulent flow can be determined using the averaged flow velocity and turbulent viscosity coefficient. To highlight this possibility, an analysis of the magnitudes of the power of the main and fluctuating components of turbulent flow is presented.

The correctness of the method is verified using an example of laminar and turbulent flows in a circular pipe. The results obtained show clearly that the proposed methodology can be used for mechanical energy loss determination in flow objects. This methodology can be used as a basis for mechanical energy loss determination in different types of flow objects.
\end{abstract}

Key words: energy decay rate, energy loss

\section{List of symbols}

C - turbulence model constant,

D - pipe diameter,

D - strain rate tensor,

$\bar{D} \quad-$ averaged strain rate tensor,

$D^{\prime} \quad-$ strain rate tensor of fluctuations,

$g \quad-$ gravitational acceleration,

$i_{0} \quad-$ longitudinal bottom slope,

$k \quad-\quad$ turbulent kinetic energy,

$L \quad-\quad$ channel or pipe length,

$l_{m} \quad-\quad$ mixing path, 


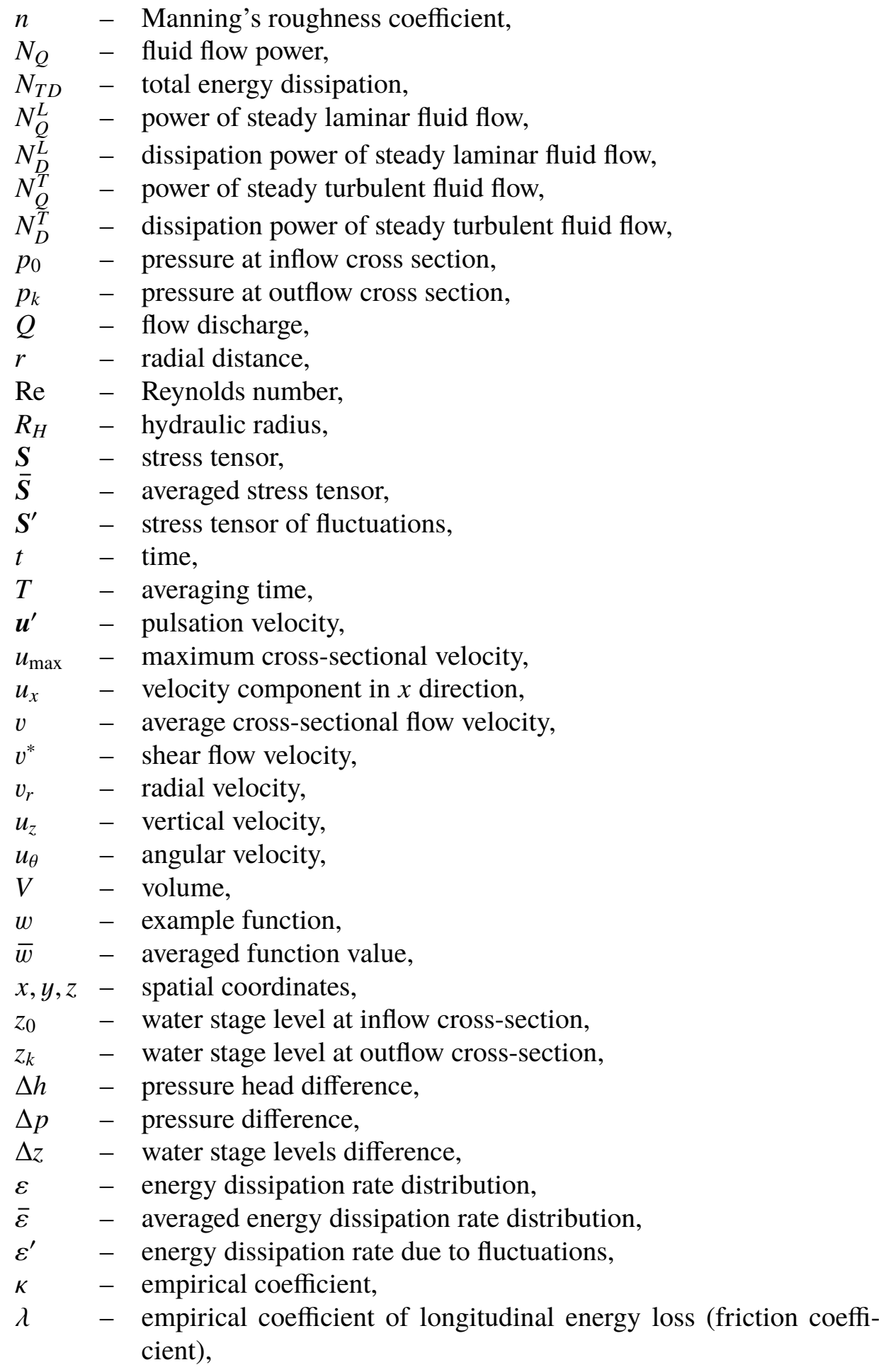




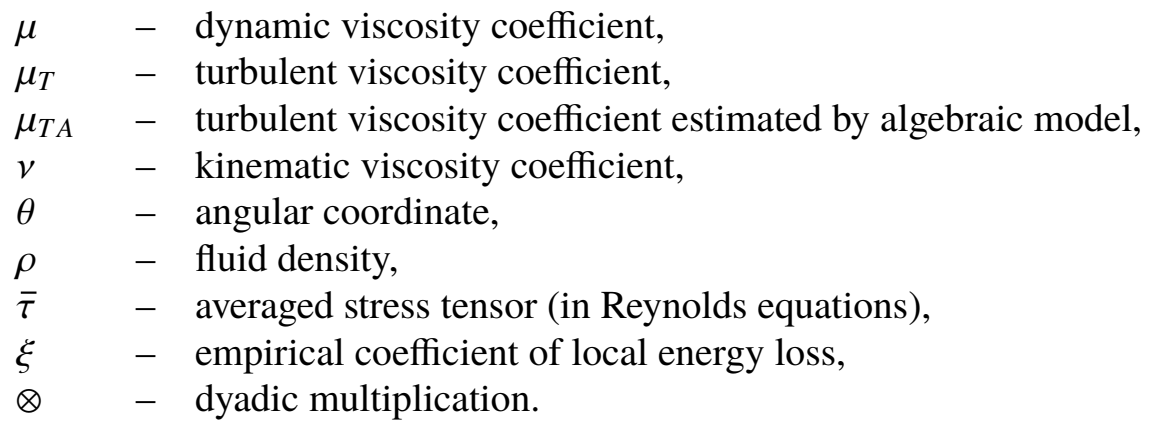

\section{Introduction}

Determination of the mechanical energy loss of the fluid flow is a significant task. This quantity is required in the design process of almost every hydraulic object. However, not only has it a practical meaning, but it also allows us to understand the physical aspects of the phenomenon occurring in the object considered.
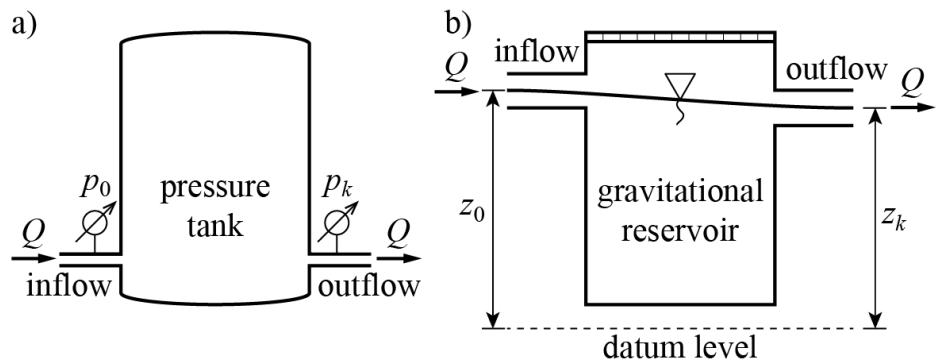

Fig. 1. Technical meaning of energy loss

The determination of energy loss is especially important in systems consisting of a single inflow and a single outflow. In pressure systems, the magnitude of energy loss is expressed as the pressure difference $\Delta p$ between the inflow pressure $p_{0}$ and the outflow pressure $p_{k}$ (Fig. 1a):

$$
\Delta p=p_{0}-p_{k} .
$$

In gravitational systems, energy loss is expressed as the function of the difference between water stage levels at the inflow cross section $z_{0}$ and the outflow cross section $z_{k}$ (Fig. 1b):

$$
\Delta z=z_{0}-z_{k} .
$$

It is also very common to use the pressure head difference $\Delta h$ to express energy loss. For pressure objects, it is expressed as

$$
\Delta h=\frac{p_{0}-p_{k}}{\rho g},
$$


whereas for gravitational systems, as

$$
\Delta h=\Delta z .
$$

From the practical point of view, the following three groups of flow systems can be distinguished in which energy loss is to be determined:

1. simple systems, which are well approximated by one-dimensional formulas,

2. compound systems of small dimensions,

3. spatially distributed systems, which often have complex three dimensional geometry.

The first category includes conduits or pipes with pressurized flow and regular open channels. Due to particularly simple characteristics of flow in such systems, it was possible to derive algebraic formulas describing energy loss. Despite the fact that these formulas have a strong empirical background, they perform very well in practice. Therefore, they are widely used in hydraulic computations (Chow 1959, French 1985, Chanson 2004, Artichowicz and Szymkiewcz 2014). The formula for energy loss estimation in the case of steady flow in circular pipes is the Darcy-Weisbach equation (Massey and Ward-Smith 1998, Puzyrewski and Sawicki 2000):

$$
\Delta h=\lambda \frac{L}{D} \frac{v^{2}}{2 g},
$$

where: $\lambda$ - empirical coefficient of longitudinal energy loss, $L$ - pipe section length, $D$ - pipe diameter, $v$ - cross-sectional average flow velocity, $g$ - gravitational acceleration.

In the case of laminar flow, the loss coefficient is expressed as

$$
\lambda=\frac{64}{\operatorname{Re}}
$$

where Re denotes the Reynolds number. In such a case, Eq. (5) becomes

$$
\Delta h=\frac{32 L v v}{g D^{2}},
$$

with $v$ denoting the kinematic viscosity coefficient.

In the case of steady uniform flow in open channels, the Manning formula can be used for mechanical energy loss estimation. In its classical form, Manning's formula (Chanson 2004, Chow 1959, Cunge et al 1979) is given by

$$
v=\frac{1}{n} R_{H}^{2 / 3} \sqrt{i_{0}},
$$

with

$$
i_{0}=\frac{\Delta h}{L},
$$


where: $n$-Manning's roughness coefficient, $i_{0}$ - bottom slope, $R_{H}$ - hydraulic radius, $L$ - channel reach length. Introduction of Eq. (9) into Eq. (8) makes it possible to estimate energy loss in a very simple manner.

In objects of the second category of flow systems, the velocity field is extremely complex. However, this category includes mostly small hydraulic fittings and measuring devices, in which, due to their small dimensions, energy loss can be estimated by a synthetic relation given by

$$
\Delta h=\xi \frac{v^{2}}{2 g},
$$

where $\xi$ denotes the empirical coefficient of local energy loss.

For systems included in the third category, the situation is complex. Large objects, such as retention reservoirs and flow reactors, require good accuracy in determination of mechanical energy loss. Here, errors can lead to significant problems in their design or functioning, which may result, for example, in liquid overflowing the edge of the reservoir. A common example of such a system is a series of reservoirs installed in waste water treatment plants. Unfortunately, there are no adequate procedures for energy loss determination tailored for such cases.

One possible solution is energy loss determination by methods of computational fluid mechanics (CFD). However, this cannot be regarded as a standard approach by engineers, as it requires expertise in differential equations and numerical methods. The problem becomes even more complicated in systems with free-surface flow, since the surface position is a function of the velocity field, which leads to the so-called Stefan problem (Vuik 1993).

In practice, technical solutions, such as "ATW-DWA Rules and Standards", are used, but their applicability is very limited because of their purely empirical formulation. In fact, they can lead to significant errors when applied to systems that differ from those for which they were developed.

In this paper, an indirect method is proposed in which mechanical energy loss is determined by a function expressing the dissipation rate of the flow energy.

\section{Determination of the Dissipation Power}

\subsection{General Considerations}

The key element of the proposed methodology is the power of energy dissipation in the fluid flow. In many hydraulic objects, the total flow power delivered to the system can be estimated using the inflow flux, which is expressed by the flow discharge $Q$ and the energy head loss $\Delta h$ :

$$
N_{Q}=\rho g Q \Delta h .
$$

In the case of steady flow, which is usually considered in technical practice, the dissipation power $N_{T D}$ is equal to the total delivered power. The difference in kinetic 
energy fluxes between inflow and outflow can be neglected, because it is usually insignificantly small, or both fluxes are identical. In the case of steady flow, the energy dissipation rate varies in space, and therefore is expressed by its density distribution $\varepsilon(x, y, z)$ (Harlow and Nakayama 1968). The total energy dissipation power in a given object is given by the following expression:

$$
N_{T D}=\int_{V} \varepsilon(x, y, z) d V,
$$

with $V$ denoting the volume and $x, y, z$ denoting spatial variables.

If $\varepsilon, V, Q$ and the fluid density $\rho$ are known, the mechanical energy loss $\Delta h$ can be determined by Eqs (11) and (12).

\subsection{Laminar Flow of Real Fluid}

Determination of the dissipation rate $\varepsilon$ is a classical element of the basic course in fluid mechanics (Badur 2009, Landau and Lifshitz 1987, Puzyrewski and Sawicki 2000, Slattery 1999). This function is expressed by the product of the viscid part of the stress tensor $\boldsymbol{S}$ and the strain rate tensor $\boldsymbol{D}$ :

$$
\varepsilon=\boldsymbol{S}: \boldsymbol{D}=\sum_{i=1}^{3} \sum_{j=1}^{3} S_{i j} D_{i j} .
$$

In the case of the real flow of a Newtonian fluid, the stress is described by

$$
S=2 \mu D,
$$

in which $\mu$ is the dynamic viscosity coefficient. Thus Eq. (13) takes the following form:

$$
\varepsilon=2 \mu \boldsymbol{D}: \boldsymbol{D} .
$$

The abovementioned relationships can be used in laminar flow only, as only for this category of flows is it possible to obtain velocity fields in explicit forms (for simple cases, in analytical forms, and for more complicated, in numerical forms).

In further considerations, a cylindrical coordinate system will be used. Eq. (15) expressed in such a system takes the following form (Slattery 1999):

$$
\begin{gathered}
\varepsilon=2 \mu\left\{\left(\frac{\partial v_{r}}{\partial r}\right)^{2}+\left[\frac{1}{2}\left(\frac{\partial v_{\theta}}{\partial \theta}+v_{r}\right)\right]^{2}+\left(\frac{\partial v_{z}}{\partial z}\right)^{2}+\frac{1}{2}\left(\frac{\partial v_{\theta}}{\partial z}+\frac{1}{r} \frac{\partial v_{z}}{\partial \theta}\right)^{2}+\right. \\
\left.+\frac{1}{2}\left(\frac{\partial v_{z}}{\partial r}+\frac{\partial v_{r}}{\partial z}\right)^{2}+\frac{1}{2}\left[\frac{1}{r} \frac{\partial v_{r}}{\partial \theta}+r \frac{\partial}{\partial r}\left(\frac{v_{\theta}}{r}\right)\right]^{2}\right\}
\end{gathered}
$$

where: $r$ - radial distance, $\theta$ - angular coordinate, $z$ - height, $v_{r}$ - radial velocity, $v_{\theta}-$ angular velocity, $v_{z}-$ vertical velocity. 


\subsection{Turbulent Flow}

To obtain the relation describing the dissipation rate of the main flow component of the turbulent flow, Eq. (13) has been rearranged according to the known methodology used in such cases. To this end, the following steps were taken:

1. Tensors were decomposed to a sum of the main part averaged with respect to time (here denoted by a horizontal bar) and the fluctuation part (here denoted by an apostrophe):

$$
\begin{gathered}
S=\bar{S}+S^{\prime}, \\
D=\bar{D}+D^{\prime} .
\end{gathered}
$$

2. Decomposed tensors were introduced into Eq. (13) and rearranged. Then both sides of the arising equation were averaged by the classical method used in turbulence theory:

$$
\bar{w}=\frac{1}{T} \int_{t-\frac{T}{2}}^{t+\frac{T}{2}} w d \tau,
$$

where: $\bar{w}$ - averaged value of the function considered, $w$ - function considered, $T$ - averaging time, $t$ - time.

3. Using basic properties of averaging (averaging for the second time does not change the value of the averaged function, the averaged value of the fluctuating quantity is equal to zero), the resulting equation was obtained:

$$
\bar{\varepsilon}=\bar{S}: \bar{D}+\overline{S^{\prime}: D^{\prime}}
$$

The values corresponding to the main part of the turbulent flow $\overline{\boldsymbol{S}}$ and $\overline{\boldsymbol{D}}$ are well defined in turbulence theory. A difficulty arises in handling the second term of Eq. (20) corresponding to the fluctuation component of the turbulent flow. According to Eq. (14), the tensor $S^{\prime}$, corresponding to the pulsation velocity of the flow of the Newtonian liquid, can be written as

$$
S^{\prime}=2 \mu D^{\prime}
$$

It means that the fluctuation term in Eq. (20) can be expressed as

$$
\overline{S^{\prime}: D^{\prime}}=2 \mu \overline{D^{\prime}: D^{\prime}} .
$$

In Eq. (22) the elements of the strain rate tensor of the fluctuation velocity $\boldsymbol{D}^{\prime}$ are local and rapidly varying functions. It is hard to estimate them on the basis of the available turbulence theory material. This material is very useful in calculating or estimating quantities related to flow, such as turbulence intensity or the kinetic energy of fluctuation. Alas, the differentiation of these functions would be burdened 
with such high uncertainty that the result would be hard to accept. Therefore, each term in Eq. (22) must be evaluated differently.

From the structural viewpoint, the situation in Eq. (22) is similar to the one which arises in deriving Reynolds equations in which the averaged stress tensor $\bar{\tau}$ consists of the Newtonian term (molecular viscosity) and the averaged dyad of the pulsation velocities $\boldsymbol{u}^{\prime}$ (Badur 2009):

$$
\bar{\tau}=2 \mu \boldsymbol{D}+\rho \overline{\boldsymbol{u}^{\prime} \otimes \boldsymbol{u}^{\prime}} .
$$

Quantitative description of the second term of Eq. (23) is a subject of advanced theory of turbulence modelling (Launder and Spalding 1972).

As a result of the dyad operator, the elements of the second term of Eq. (23) are squared. Apart from the boundary layer, where the influence of molecular viscosity becomes significant, the second term of Eq. (23) plays an extremely important role in the formation of the velocity profile.

However, the problem considered here is slightly different because the pulsation term in Eq. (20) is included globally through the integral given by Eq. (12).

Note that the right-hand side of Eq. (22) is the rate of kinetic energy dissipation $\varepsilon^{\prime}$ multiplied by the fluid density (Launder and Spalding 1972). It is a quantity playing a great role in turbulence modelling. For example, it is a basis of the very popular " $k-\varepsilon$ " turbulence model, according to which the turbulent viscosity coefficient can be expressed as (Tennekes and Lumley1972):

$$
\mu_{T}=C \frac{k^{2}}{\varepsilon^{\prime}}
$$

with $k$-turbulent kinetic energy, $C$ - model constant, determined as $C=0.09$ and $\varepsilon^{\prime}$ - energy dissipation rate due to fluctuations.

This offers the possibility of estimating the $\varepsilon^{\prime}$ value and comparing it with the first term of the right-hand side of Eq. (20). To this end, let us highlight the fact that different turbulence models widely accepted in the field (Bardina et al 1997) give similar values of the $\mu_{T}$ coefficient. As a comparative relationship, let us use the formally simplest algebraic model for estimation of $\mu_{T}$ (Launder and Spalding 1972):

$$
\mu_{T A}=0.0168 \rho v^{*} l_{m},
$$

in which the shear flow velocity $v^{*}$ and the mixing path $l_{m}$ are expressed by simple standard relationships (ATV-DWA 2000):

$$
\begin{gathered}
v^{*}=\sqrt{\frac{\lambda}{8}} v, \\
l_{m}=\kappa R=0.41 R,
\end{gathered}
$$

where: $\kappa$-empirical coefficient, $R$ - pipe radius, $\lambda$-empirical friction coefficient. 
Assuming additionally that the average fluctuation intensity rate is about $4 \%$ of the mean flow velocity (Prandtl 1956), it can be written that

$$
l_{m}=0.0016 v^{2} .
$$

Comparing Eqs (24) and (25) and taking into consideration Eqs (26) and (27), the following approximated relation is obtained:

$$
\rho \cdot \varepsilon^{\prime}=0.000092 \frac{\rho v^{3}}{\sqrt{\lambda} R} .
$$

The value of the first term on the right-hand side of Eq. (20) can be estimated using Eqs. (2) and (8). Referring the power of the main flow to the unit volume of the fluid in the circular pipe,

$$
\frac{N_{Q}}{V}=\frac{\rho \lambda v^{3}}{4 R}
$$

with $V=\pi R^{2} L$.

Dividing both sides of Eq. (30) by Eq. (29), the following expression is obtained:

$$
\frac{N_{Q}}{V \rho \varepsilon^{\prime}}=2706 \lambda^{3 / 2}
$$

Eq. (31) shows the ratio of the main flow power to the power of turbulent fluctuations in the circular pipe. It is worth noting the structural similarity of Eqs (29) and (30), due to which Eq. (31) does not contain specific geometrical characteristics of the given system. Instead it contains only Nikuradse's friction coefficient $\lambda$. Introducing its typical average value of order $\lambda=0.04$ into Eq. (31), the following expression is obtained:

$$
\frac{N_{Q}}{V \rho \varepsilon^{\prime}}=21.6
$$

Eq. (32) shows that the main flow power is several dozen times the power of the fluctuations. It leads to the conclusion that the second term on the right-hand side of Eq. (20) can be rejected, which yields:

$$
\bar{\varepsilon}=\overline{\boldsymbol{S}}: \overline{\boldsymbol{D}}=2 \mu_{T} \overline{\boldsymbol{D}}: \overline{\boldsymbol{D}} .
$$

The exact realizations of this expression are obtained by referring to the definition of the averaged strain rate tensor. For a cylindrical coordinate system it will result in an expression similar to Eq. (16), in which the velocity components will be replaced by the averaged ones, and the molecular viscosity coefficient $\mu$ will be replaced by the turbulent viscosity coefficient $\mu_{T}$. 


\section{Example Applications of the Proposed Method}

\subsection{Laminar Flow in a Circular Pipe}

Let us consider a flow of discharge $Q$ in a circular pipe of diameter $D=2 R$ and length $L$. During laminar flow, the power $N_{Q}$ necessary to maintain the flow in the technical approach (using energy head loss) is described by the simple algebraic relations mentioned above. Introducing Eq. (7) into Eq. (11) and expressing the average flow velocity $v$ by means of the flow discharge $Q$ and the pipe diameter $D$, the following expression is obtained:

$$
N_{Q}^{L}=\frac{128 \mu L Q^{2}}{\pi D^{4}} .
$$

In the "hydro-mechanical" approach, the dissipation power $N_{D}$ (which in the case of steady flow is equal to the power of the flow) is expressed by Eqs (12) and (16). In the case of laminar flow in a circular pipe, Eq. (13) yields

$$
\varepsilon=\mu\left(\frac{\partial u_{x}}{\partial r}\right)^{2} .
$$

The velocity field is given by the classical relation (Massey and Ward-Smith 1998):

$$
u_{x}(r)=\frac{\Delta p}{4 \mu L}\left(R^{2}-r^{2}\right)=\frac{32 Q}{\pi D^{4}}\left(R^{2}-r^{2}\right) .
$$

Introducing Eq. (36) into Eq. (35), one obtains

$$
\varepsilon=\frac{4096 Q^{2} r^{2} \mu}{\pi^{2} D^{8}}
$$

Integrating Eq. (37) in the whole volume of the pipe of diameter $D$ and length $L$, one obtains

$$
N_{D}^{L}=\frac{128 \mu L Q^{2}}{\pi D^{4}} .
$$

The result is consistent with obvious expectations: the dissipation power in laminar flow $N_{D}^{L}$ is equal to the power lost during flow $N_{Q}^{L}$ (Eq. (34)).

\subsection{Turbulent Flow in a Circular Pipe}

In the case of laminar flow, the identical expression for $N_{Q}^{L}$ and $N_{D}^{L}$ is a matter of course. This result could be obtained because of the existence of analytical expressions for the velocity field. A more interesting case is the turbulent flow, since the flow description is not unequivocal, but depends on the choice of the turbulence model.

Determination of the power of the flow is still a trivial task, which gives

$$
N_{Q}^{T}=\frac{8 \rho \lambda L Q^{3}}{\pi^{2} D^{5}} .
$$


To obtain the value $N_{D}^{T}$ in an algebraic form that can be compared with $N_{Q}^{T}$, a very simple relation describing the flow profile was chosen. In this model, the velocity field is described by the relationship proposed over 150 years ago by Darcy (1857). Let us express this relation in a form containing shear velocity (Lojcjanskij 1973):

$$
\frac{u_{\max }-u(r)}{v^{*}}=5.08\left(\frac{r}{R}\right)^{3 / 2} .
$$

This makes it possible to determine the required derivative

$$
\frac{\partial u}{\partial r}=-\frac{7.62 \cdot v^{*}}{R}\left(\frac{r}{R}\right)^{1 / 2} .
$$

This time, the turbulent viscosity coefficient $\mu_{T}$ was estimated by the Prandtl model (Prandtl 1956), which is a slightly more complex formula than the one used previously (Eq. (25)):

$$
\mu_{T}=\kappa^{2} \rho R^{2}\left|\frac{\partial u_{x}}{\partial r}\right| .
$$

Introducing Eqs (40) and (41) into Eq. (33) and integrating it according to Eq. (12) throughout the whole volume of the pipe, one obtains:

$$
N_{D}^{T}=60.1 \frac{\rho \lambda^{3 / 2} Q^{3} L}{\pi^{2} D^{5}} .
$$

Comparison of Eqs (39) and (42) shows that, in the case of turbulent flow, the power of flow and the power of dissipation are not identical, unlike in laminar flow. However, both relationships have an almost identical formal structure, and their ratio is a function of longitudinal loss only:

$$
\frac{N_{D}^{T}}{N_{Q}^{T}}=7.52 \sqrt{\lambda} .
$$

A similar type of relationship is obtained in considerations resulting with Eq. (31). The non-identity of Eqs (39) and (42) is entirely understandable, since the turbulence model used was very simple, as was the velocity profile. However, the result of Eq. (43) does not deviate much from unity. In a range of practical variability of the loss coefficient $\lambda \in[0.01,0.08]$ (according to the Nikuradse diagram), it changes in the range from 0.752 to 2.127 (Fig. 2).

\section{Conclusions}

The dissipation power of flowing fluid is a component of the equation of its internal energy balance. Moreover, as a standalone quantity, it contains significant information about the flow phenomenon considered. It can be used as an auxiliary relationship 


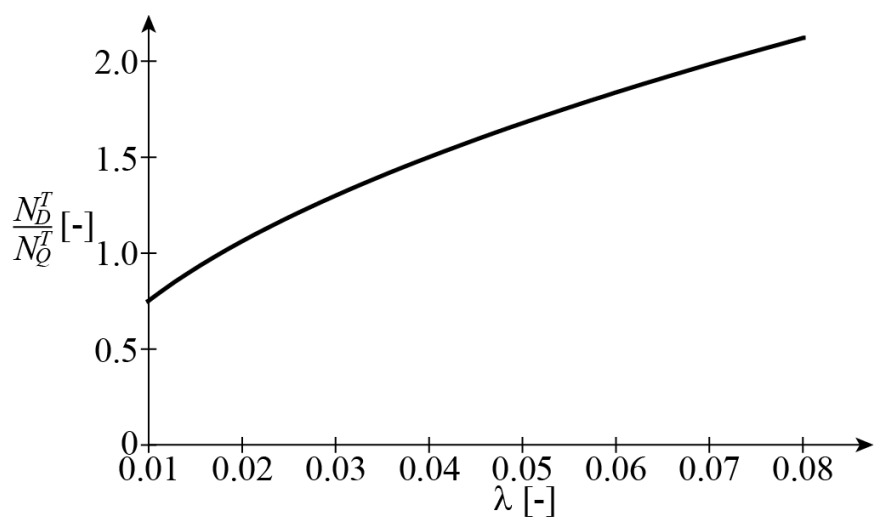

Fig. 2. Simplified model of turbulent flow in a circular pipe: the ratio of the dissipation power to the flow power

in CFD methods, but it can also be an independent element in simplified fluid flow description methods.

In its original form, it can be applied to laminar flows. In practice, however, the most interesting are turbulent flows.

In this work, it has been shown that the dissipation intensity rate of the fluid flow is predominantly used to sustain the main flow. The energy loss induced by turbulent fluctuations of velocity is only a small percentage of the total energy loss. This estimation makes it possible to directly apply Eq. (15) to the averaged velocity, with the turbulent viscosity coefficient given by Eq. (41).

The results obtained were applied to steady flow in a circular pipe. The simple geometry of that system makes it possible to obtain algebraic relationships. In the case of laminar flow, it turned out that relations describing energy loss expressed by the energy dissipation rate and relations using the technical approach are the same.

In the case of turbulent flow, the relationships obtained are almost identical formally and very similar quantitatively (Eqs (43), Fig. 2).

The results presented in this work are a proper starting point to further research on energy loss estimation in more complex objects.

\section{References}

Artichowicz W., Szymkiewicz R. (2014) Computational issues of solving the 1D steady gradually varied flow equation, J. Hydrol. Hydromech., 62 (3), 226Ő-233, DOI: 10.2478/johh-2014-0031.

ATV-DWA rules and standards (2000) http://en.dwa.de/ 2017.

Badur J. (2009) The development of the energy concept (in Polish), IMP PAN, Gdańsk.

Bardina J. E., Huang P. G., Coakley T. J. (1997) Turbulence Modeling Validation, Testing, and Development, NASA Technical Memorandum 110446.

Chanson H. (2004) The Hydraulics of Open Channel Flow: An Introduction, Second Edition, Elsevier, Oxford.

Chow V. T. (1959) Open-channel Hydraulics, McGraw-Hill/Kogakusha Company LTD, Tokyo. 
Cunge J. A., Holly F. M., Verwey A. (1979) Practical Aspects of Computational River Hydraulics, Pitman, London.

Darcy H. (1857) Recherches experimentales relatives an mouvement de l'eaux daus les tuyaux, Mallet-Bachelier, Paris.

French R. H. (1985) Open Channel Hydraulics, McGraw-Hill, New York.

Harlow F. H., Nakayama P. T. (1968) Transport of Turbulence Energy Decay Rate, Los Alamos Scientific laboratory, Report LA-3854, Univeristy of California.

Landau L. D., Lifshitz E. M. (1987) Fluid Mechanics, Second Edition (Course of Theoretical Physics), Elsevier Butterworth Heinemann, Oxford.

Launder B. E., Spalding D. B. (1972) Lectures in Mathematical Models of Turbulence, Academic Press, New York-London.

Lojcjanskij Ł. G. (1973) Mechanics of Liquids and Gases (in Russian), Nauka, Moscow.

Massey B., Ward-Smith J. (1998) Mechanics of Fluids, Stanley Thornes Ltd. Cheltenham, UK.

Prandtl L. (1956) Dynamics of Flows (Polish translation), PWN, Warsaw.

Puzyrewski R., Sawicki J. M. (2000) Fundamentals of Fluid Mechanics and Hydraulics (in Polish), $3^{\text {rd }}$ ed., PWN, Warsaw.

Slattery J. C. (1999) Advanced Transport Phenomena, University Press, Cambridge, UK.

Tennekes H., Lumley J. T. (1972) A First Course in Turbulence, MIT Press, Cambridge, USA.

Vuik C. (1993) Some historical notes on the Stefan problem, Nieuw. Archief voor Wiskunde, 4e serie, 11 (2), 157-167. 